\title{
Reflexión de la humanización de la atención: teoría de Jean Watson y propuesta de su aplicación
}

Autores:

Miguel Andrés Valencia Contrera. Magíster ๑ en Enfermería. Universidad de Concepción. Mail: m.valencia@outlook.cl Angélica Melita Rodríguez. Doctora en Enfermería. Universidad de Concepción. Mail: amelita@udec.cl

Fecha de Recepción: 08 de Marzo de 2021 Fecha Aceptación: 27 de Septiembre de 2021 DOI: http://doi.org/10.22370/bre.61.2021.3037

\section{> Resumen}

Introducción: Con el paso del tiempo la atención en salud se ha caracterizado por prácticas que hacen falta a la ética profesional, cuyos cimientos parecieran ser copiosos y con disímiles características; la teoría del cuidado humanizado de Jean Watson proporciona una forma de tratar la problemática. Objetivo: Describir las piedras angulares del trabajo de Jean Watson y proporcionar una propuesta de aplicación que entregue respuesta a las actuales necesidades de humanización del cuidado y de la atención en salud de la población chilena. Metodología: Artículo descriptivo-analítico, el cual se organizó en dos etapas, en la primera de ellas se realizó una revisión descriptiva incluyendo libros, producciones científicas, entrevistas, material audiovisual y se complementó con una revisión del estado del arte en la base de datos Web of Science y en una segunda etapa se expone una propuesta de aplicación teórica que permita el abordaje del cuidado humanizado. Conclusiones: Se logró describir los elementos transversales del trabajo de Jean Watson, la que fundamenta la propuesta de generar normas o protocolos donde se desarrolle una estandarización de la humanización del cuidado humano, cuyos resultados sean aquilatados a través de indicadores de cuidados humanizados.

> Palabras claves: Enfermería, humanización de la atención, filosofía en enfermería, atención de enfermería, teoría de enfermería (DeCS - BIREME). 


\section{Reflection on the}

\section{humanization of care:}

\section{Jean Watson's theory}

and proposal for its

application

\section{> Abstract}

Introduction: With the passage of time, health care has been characterized by practices that are lacking in professional ethics, whose foundations of the problem seem to be copious and with dissimilar characteristics, Jean Watson's theory of humanized care provides us with a way of treating the problem. Objective: to describe the cornerstones of Jean Watson's work, and to provide an application proposal that responds to the current needs of humanization of care and health care of the Chilean population. Methodology: Descriptive-analytical article, which was organized in two stages, in the first of them a descriptive review was carried out including books, scientific productions, interviews, audiovisual material and was complemented with a review of the state of the art in the database Web of Science, and in a second stage a proposal of theoretical application that allows the approach of humanized care is presented. Conclusions: it was possible to describe the transversal elements of Jean Watson's work, which bases the proposal to generate norms or protocols where a standardization of the humanization of human care is developed, the results of which are assessed through humanized care indicators.

> Keywords: Nursing, humanization of assistance, nursing philosophy, nursing care, nursing theory (DeCS- BIREME). 


\section{) Introducción:}

Con el paso del tiempo la atención en salud se ha caracterizado por prácticas que hacen falta a la ética profesional, cuyos cimientos del problema parecieran ser copiosos y con disímiles características (1); ante ello, los pacientes poco a poco se han empoderado y se hacen escuchar más, exigiendo sus derechos (2).

Noticias como: “Usuarios de urgencia maternal de Hospital de Puerto Montt denuncian mala atención y maltratos" (3), "Mujer acusa malos tratos en Cesfam de Temuco: reclamó por larga espera y llamaron a Carabineros" (4), "Uno de cada cuatro reclamos en hospitales y consultorios públicos es por maltrato" (5), "Denuncian malos tratos a niño con autismo en cesfam de Calle Larga" (6), "Ustedes no se vacunan: acusan malos tratos a pareja de adultos mayores en cesfam de Concepción" (7), "Decide no operarme hasta que llegue el cheque en garantía: doctor deberá pagar millonaria indemnización" (8), "Joven denunció negligencia médica tras ser operado del pie equivocado" (9), "Dijeron que me había cagado la vida: violencia obstétrica" (10); dejan al descubierto la necesidad referida de humanizar el cuidado, pareciera increíble tener que enfatizar en humanizar algo que su existencia responde a una necesidad humana, los profesionales de la salud trabajan con personas, y qué más humano que ayudar a otro en su dolencia.

Es por ello, que dichos antecedentes generan un llamado de atención a todos los profesionales que practican el arte de cuidar, y no solo enfermería como estamento que cumple un rol protagónico a la hora de hablar de profesionales capacitados para ser frente al problema, pues son estos últimos, los encargados de la gestión del cuidado, como rol exclusivo y excluyente en el territorio chileno. De esta forma dar cumplimiento a los valores- que según Watson- enfermería posee (11):
“Una profesión que pierde sus valores es una profesión sin corazón, una profesión sin corazón es una profesión sin alma, y una profesión sin alma ni corazón, es una profesión innecesaria".

Se destaca la impersonalidad en el trato de los pacientes como un elemento crucial que da a luz al fenómeno en cuestión (12), de éste a su vez surge el término ampliamente utilizado: "deshumanización del cuidado o de la atención en salud".

Bajo la anterior premisa, se gesta el presente manuscrito que responde al objetivo de describir las piedras angulares del trabajo de Jean Watson, y proporcionar una propuesta de aplicación, que dé respuesta a las actuales necesidades de humanización del cuidado y de la atención en salud de la población chilena.

\section{> Metodología:}

Artículo descriptivo-analítico, basado en el aporte teórico del Cuidado Humanizado de Jean Watson, a partir de su análisis en profundidad se proporciona y se fundamenta una propuesta de aplicación que facilite la entrega de cuidados humanizados en la práctica asistencial; para tal efecto, el artículo se organizó en dos etapas:

La primera etapa consistió en la realización de una revisión descriptiva exhaustiva sobre el aporte de la teorista, incluyendo: libros, producciones científicas, entrevistas y material audiovisual donde la autora explica su contribución; sumado a esto, para enriquecer la información se complementó con una visión externa de su trabajo, con estudios que dan cuenta de su uso en la práctica, para ello se realizó una revisión del estado del arte, en la base de dato Web of Science (WOS), considerando como criterios de inclusión aquellos artículos que presenten un máximo de 5 años de antigüedad (periodo 2015-2020), que contuvieran en su 
título la palabra "Watson" y que incluyeran a Jean Watson como referente teórico. Se eliminan duplicados. Finalmente se incorporaron 27 documentos que formaron parte del cuerpo de la reflexión: 2 documentos del Watson Caring Science Institute (13,38), 9 libros (14-16,18,19,22,24-26), 11 artículos de investigación $(21,23,28-31,33,35-37,39)$ y 5 tesis $(17,20,27,32,34)$.

La segunda etapa consistió en exponer una propuesta de aplicación del marco teórico proporcionado por Jean Watson, que permita el abordaje del cuidado humanizado en el quehacer de la enfermería chilena.

\section{) Resultados}

\section{Hitos y antecedentes de la Teoría de Jean Watson}

Margaret Jean Harman Watson, asistió a la Escuela de Enfermería Lewis Gale en Roanoke, Virginia; se graduó en 1961, continuó su educación en la Universidad de Colorado, obtuvo su licenciatura en enfermería en 1964, una maestría en 1966 y un doctorado en psicología educativa y asesoramiento en $1973(13,14)$.

El seguimiento de sus creaciones literarias refleja la evolución de su teoría del cuidado: su primer libro, Enfermería la filosofía y la ciencia del cuidado, publicado en 1979 (15), los 11 factores curativos de Yalom estimularon a Watson a usar 10 factores cautelares como marco organizativo para su libro, documento que da indicios de aquellos elementos indispensables que enfermería debería tener en su quehacer, más tarde los operacionaliza para facilitar su comprensión, poniendo en manifiesto la importancia del cuidado humanizado (16).

Su segundo libro, Enfermería: ciencia humana y cuidado humano - una teoría de enfermería (1985), abordó sus problemas conceptuales y filosóficos en enfermería (16).
Su tercer libro, Enfermería postmoderna y más allá (1999), presentó un modelo para llevar la práctica de enfermería al siglo XXI. Watson describe dos eventos personales que alteran su vida contribuyendo a su escritura: en 1997 experimentó un accidente dental que resultó en la pérdida de su ojo izquierdo, y tiempo después, en 1998, su esposo murió, ella refiere "intenté integrar estas heridas en mi vida y mi trabajo. Unos de los regalos a través del sufrimiento fue el privilegio de percibir y recibir mi propia teoría a través del cuidado de mi esposo y amables enfermeras, amigos y colegas" (16).

Instrumentos para evaluar y medir el cuidado en enfermería y ciencias de la salud (2002), su cuarto libro, el cual comprende una colección de 21 instrumentos para evaluar y medir el cuidado (14), libro que tributa a la mejora de la práctica diaria, es sabido que de la práctica se genera nuevo conocimiento, mediciones del cuidado que denotan una realidad o fenómeno, en su intento de comprenderlo se integra la teoría, haciendo fusión la triada práctica-teoría-investigación.

Su quinto libro "cuidar la ciencia como ciencia sagrada (2005)", describe su viaje personal para mejorar la comprensión sobre la ciencia solidaria, práctica espiritual, el concepto y la práctica de cuidado y cuidado-trabajo curación, en este libro ella guía al lector a través de experiencias que invitan a la reflexión y lo sagrado de enfermería enfatizando una profunda reflexión interna y personal de crecimiento, habilidades de comunicación, uso del crecimiento autotranspersonal, y la atención a la ciencia del cuidado y la curación a través de entrega, gratitud y rendición (14).

Sus libros recientes incluyen medición del cuidado: Investigación Internacional sobre Caritas como Curativa; creación de un plan de estudios de ciencia solidaria; la ciencia del cuidado humano: 
Una teoría de la enfermería; y ciencia cuidadosa, práctica consciente: Implementando la teoría del cuidado humano de Watson (16).

\section{Proceso caritas, comprendiendo el proceso de vida de las experiencias humanas}

Jean Watson en su libro Enfermería: La filosofía y la ciencia del cuidado (1979) proporcionó el núcleo y estructura original para la Teoría del Cuidado Humano: Diez Factores Caritativos; dichos factores fueron identificados como los aspectos esenciales del cuidado en enfermería, sin el cual los enfermeros y enfermeras podrían no haber estado practicando enfermería profesional. Su trabajo con los procesos caritas ha perdurado en el tiempo, sin sufrir modificaciones desde su publicación original (16).

Watson propone los 10 Factores de Cuidados que después trasladó a Proceso Caritas de Cuidados, corresponde a una extensión de los anteriores, pero ofrece un lenguaje más fluido, para entender en un nivel más profundo las dimensiones del proceso de vida de las experiencias humanas, para facilitar la comprensión de los cuidados de enfermería como la ciencia del cuidar (17).

El término caritas según Watson se utiliza en contraste con el término curativo para ayudar al estudiante de enfermería a diferenciar la enfermería, de la medicina. Caritas significa en latín: valorar, apreciar, prestar atención especial, atención afectuosa. Los procesos caritas incluyen una dimensión decididamente espiritual y una vocación manifiesta al amor y al cuidado (18); estos factores son los que enfermería utiliza en la prestación de la atención hacia el paciente, los cuales fueron desarrollados a partir de una filosofía humanística. Según Watson estos factores no son factores determinados como "finales", pues puede existir un número ilimitado de formas de caracterizar y producir un resultado terapéutico en el cuidado de enfermería (19).

\section{Supuestos básicos de la Teoría de Jean Watson}

Los supuestos, son premisas asumidas como verdad sin comprobación, los supuestos de Watson se enmarcan en la espiritualidad de la vida, las facultades de adaptación humanas que permiten crecer y cambiar, el respeto y aprecio por la persona y la vida, la libertad para tomar decisiones y la importancia de una relación enfermero/a-paciente basada en sentimientos y emociones que permitan una cognición compartida y en consenso (20).

Los siguientes son los supuestos básicos para la ciencia del cuidado de enfermería según Watson (16): $N^{\circ} 1$ el cuidado solo puede demostrarse y practicarse efectivamente interpersonalemente; $\mathrm{N}^{\circ} 2$ El cuidado consiste en factores cautelares que resultan en la satisfacción de ciertas necesidades humanas; $\mathrm{N}^{\circ} 3 \mathrm{El}$ cuidado efectivo promueve la salud y el crecimiento individual o familiar; $\mathrm{N}^{\circ} 4$ Las respuestas afectuosas aceptan a una persona no solo como son ahora, sino como lo que él o ella pueden llegar a ser; $\mathrm{N}^{\circ} 5$ Un ambiente de cuidado es aquel que ofrece desarrollo de potencial mientras le permite a la persona elegir la mejor acción para sí misma, en un momento dado; Nº El cuidado es más saludable que el curar. La práctica del cuidado integra el conocimiento biofísico con conocimiento del comportamiento humano para generar o promover salud y proporcionar servicios a quienes están enfermos. Por lo tanto, una ciencia del cuidado es complementaria a la ciencia de curar; $\mathrm{N}^{\circ} 7$ La práctica del cuidado es fundamental para la enfermería.

Hay 10 factores caritativos primarios que forman una estructura para estudiar y entender la enfermería como la ciencia del cuidado, algunos de es- 
tos, según lo referido por Watson (14) son de difícil comprensión, y por ende una limitante en su posterior aplicación, por ello los modificó y se gestaron los 10 procesos caritas $(15,21)$.

\section{Metaparadigmas del trabajo de Jean Watson}

Watson en su libro ciencia del cuidado humano: una teoría de enfermería, publicado en el año 2012 (21) refiere que su teoría no es una "teoría científica dura" sino que sigue siendo una teoría; dicha teoría ha sido mencionada como un marco, como un modelo conceptual, como una filosofía, como una teoría de rango medio, y también puede clasificarse como de introducción, ya que se basa en el trabajo de diferentes autores y su propia investigación en la práctica y la educación (23).

En cuanto al metaparadigma enfermero, según Watson la representación de enfermería, salud, persona y entorno indica que existe una apertura entre cada uno de los elementos del metaparadigma, entre los conceptos básicos y las interacciones entre todas las partes de la teoría (24); persona definida como "un ser espiritual en el mundo", salud como "unidad y armonía dentro de la mente, el cuerpo y el alma", entorno definido implícitamente como "ambiente mental, físico, social y espiritual de apoyo, protección y/o correctivo", enfermería como "una ciencia humana de personas y experiencias de salud humana-enfermedad mediadas por transacciones profesionales, personales, científicas, estéticas y éticas de cuidado humano" $(25,26)$.

La descripción de la teoría del arte es diferente de la de otras teorías, ya que enfatiza una comprensión profunda de sí mismo y las relaciones transpersonales de persona a persona para mantener la armonía interna de la mente, el cuerpo y especialmente el alma (19). Es por ello que Watson refiere que una persona se enferma cuando hay falta de armonía dentro de la mente, el cuerpo y el alma, siendo más abstracto en sus postulados "la enfermedad no es necesariamente una enfermedad", más bien, es una agitación de desarmonía consciente o inconsciente dentro de una persona (25).

En lo referente a los fenómenos Watson desarrolló la teoría en torno a los fenómenos de la experiencia de salud-enfermedad humana y las experiencias de cuidado y curación de persona a persona (22). En cuanto a los conceptos, según Watson son la relación de cuidado transpersonal, los momentos de cuidado/ocasiones de cuidado, la consciencia de cuidado y los procesos caritas. El concepto de una relación de cuidado transpersonal tiene tres dimensiones: el yo, el campo fenomenológico y la intersubjetividad. Los conceptos de momento cariñoso/ocasión cariñosa y consciencia cariñosa son unidimensionales (23).

\section{Aplicación de la Teoría de Jean Watson a la prácti- ca enfermera}

Según lo expuesto por Pajnkihar et al (23), la teoría de Watson ofrece grandes beneficios para la enfermería, ello evidenciado en muchos países en el desarrollo de relaciones de cuidado transpersonales y un énfasis en la mente, el cuerpo y el alma del individuo; ello debido a que alienta a practicar el cuidado humano, sacando a la luz su objetivo de respetar la integridad, la dignidad, además de aplicar conceptos teóricos, cultivar momentos de cuidado a través de la práctica de los procesos caritas.

En la tabla №1 se exponen algunos escenarios donde se ha utilizado la teoría de Jean Watson, siendo categorizada cada investigación según título del artículo, autor/res, país, año y aplicación de la teoría. 
TABLA 1: ESTUDIOS IDENTIFICADOS QUE HAN APLICADO LA TEORÍA DE JEAN WATSON

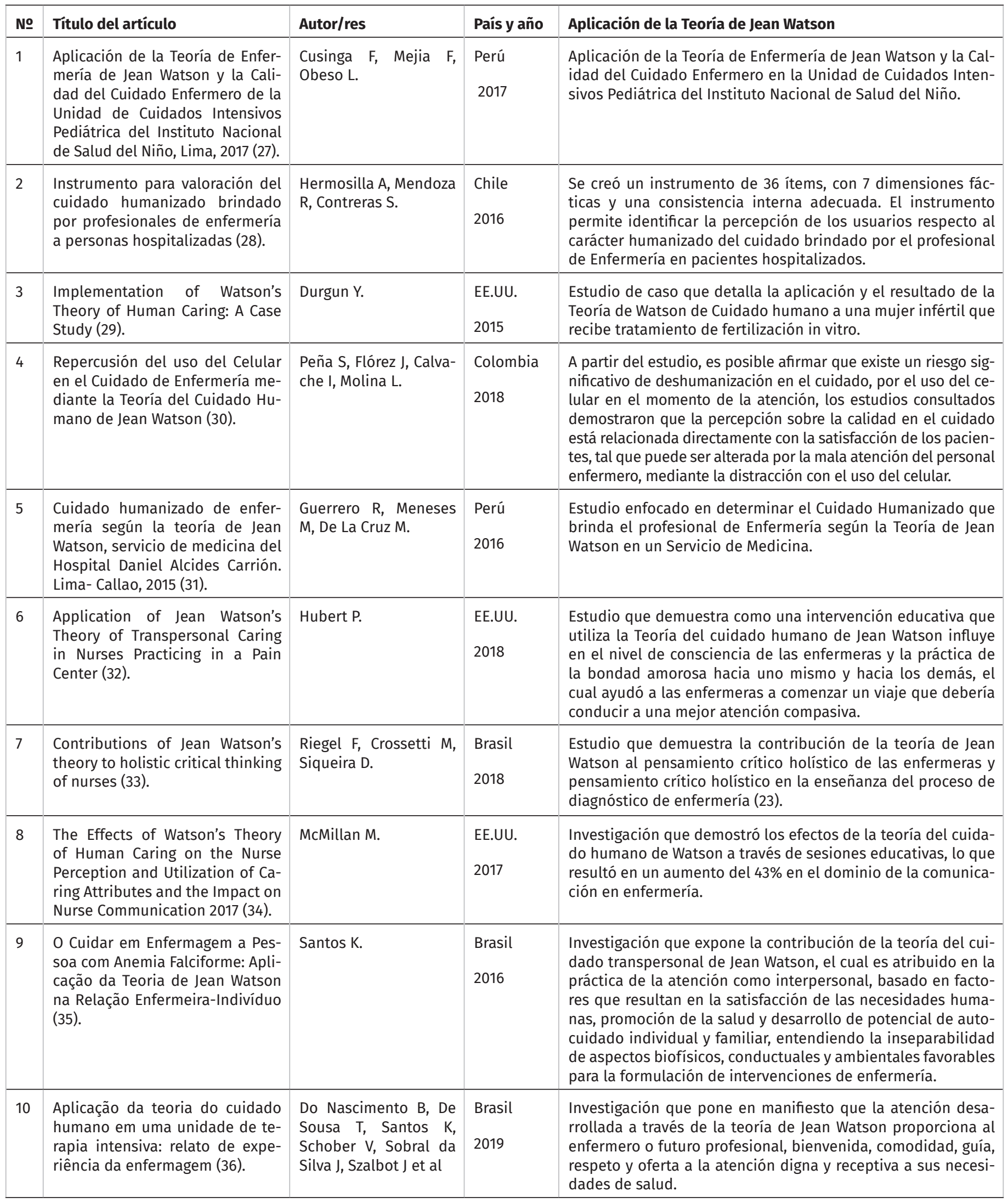

Fuente: Elaboración propia 
En razón de lo expuesto, ha quedado en evidencia que son múltiples los escenarios donde puede ser aplicada la teoría del cuidado humano de Jean Watson; abarcando las cuatro funciones que posee enfermería (asistencia, educación, administración e investigación) para dar cumplimiento a su rol, el de "cuidar".

En cuanto al área asistencial, la teoría de Watson se puede aplicar a varias poblaciones y en diferentes entornos clínicos según lo expuesto en la tabla 1, por ejemplo: en una Unidad de Cuidado Intensivos Pediátrica (27) o en el Cuidado humano a una mujer infértil que recibe tratamiento de fertilización (29); según lo expuesto por Seaward et al (37), es imperativo que los enfermeros comprendan la visión del mundo de sus pacientes, y esto incluye su perspectiva espiritual para la mejor atención posible. Ello responde a la necesidad y creatividad que cada enfermero desee proyectar en su práctica.

En lo referente a la investigación, a nivel mundial, la teoría de Watson se ha utilizado como marco de investigación en muchos estudios diferentes; por ejemplo, la base de datos comparativa internacional Watson Caritas y la puntuación del paciente Watson Caritas (WCPS) desarrollaron un instrumento con cinco preguntas de cuidado, críticas para evaluar las prácticas de cuidado y la satisfacción del paciente. Esto representa la investigación clínica multisitio en sistemas que utilizan la teoría de Watson (38). Además, puede ser evidenciado su uso en la investigación en los esfuerzos por utilizar la teoría de Watson en nuevos horizontes, al surgir la necesidad de humanizar la atención.

En lo referente a la función educativa de enfermería, la teoría de Watson se ha utilizado en numerosos planes de estudios de enfermería, estos responden a la necesidad de una práctica profesional de educación específica de la disciplina, y que esté guiada por valores basados en la ciencia del cuidado humano; existen estudios que demuestran cambios objetivables post formación en la teoría de Jean Watson, por ejemplo: participantes que completaron el Programa de Educación para Entrenadores de Caritas, los cuales se evaluaron con pretest-postest para evaluar los cambios en las percepciones de autocuidado, liderazgo de caritas y comportamientos de los compañeros de trabajo, después de completar el Programa de Educación para Entrenadores de Caritas, cuyos resultados de las puntuaciones medias de todas las medidas mejoraron significativamente (39).

Finalmente, la función administrativa de enfermería, en donde su uso se encuentra asociado a la gestión de recursos humanos de enfermería, como también en sistemas computarizados de documentación clínica en ocho organizaciones hospitalarias (23).

\section{Propuesta teórica para la aplicación de la Teoría de Jean Watson al quehacer de enfermería.}

Con el propósito de orientar a los profesionales de la salud en la toma de decisiones clínicas, ya sea en aspectos prácticos o administrativos, es que hoy en día existen una gran cantidad de normas y protocolos en las instituciones de salud (40); por lo general, estas suelen tener un enfoque biomédico, vale decir, se enfocan en guiar o regular procedimientos, técnicas, manejo de patologías y/o prevención de las mismas, ello sujeto a la necesidad de cada Unidad, Servicio y/o Establecimiento.

Bajo la anterior premisa, es que se gesta la presente propuesta, enfocada en la carencia de orientación, guía y/o regulación del cuidado humanizado. Para poder germinar la presente propuesta, es imperativo preguntarnos ¿qué sucede cuando los profesionales se enfrentan a situaciones que ameritan intervenciones de contenciones emocio- 
nales o cuidados humanizados?, ¿suele ser la humanización de la atención un foco predominante en la estandarización del cuidado?

La respuesta utópica a dichas interrogantes sería: que el abordaje de la atención en salud es humanizado, cálido, cauto y con buenos indicadores de satisfacción usuaria, no obstante, no es la realidad en la práctica, son numerosos los casos documentados (8-15) donde existe una debacle en la atención, son copiosas las situaciones donde se deja en evidencia una escasa humanización de lo que deber serlo por su naturaleza "una atención en salud".

Si se protocoliza o normaliza un procedimiento biomédico para dar cumplimiento a indicadores en salud, ¿por qué no hacer protocolos y normas que den cumplimiento a indicadores de humanización del cuidado?, si bien es cierto, que en la actualidad escasean los estudios que nos proporcionen dichos indicadores debido a la complejidad de la temática, sin embargo, existen esfuerzos que intentan atender dichas necesidades en salud, por ejemplo, un estudio realizado en contexto de una tesis doctoral en enfermería denominada "Indicadores de cuidado humanizado de enfermería en hospitales de la región del Bio Bío, Chile" (41), el cual es un excelente cimiento para la generación de dichas normas o protocolos. Es ahí donde se enmarca esta propuesta: la de crear normas o protocolos donde se desarrolle una estandarización de la humanización del cuidado humano, y cuyos resultados sean aquilatados a través de indicadores de cuidado humanizados, es una propuesta un tanto ambiciosa, no obstante, existe información suficiente que avala su aplicación.

Bajo la problemática expuesta, se hace un llamado a su concientización, los presentes autores manifiestan el carácter perentorio del abordaje del cuidado humanizado, a través de la creación de normas o protocolos, que logren concebir la estandarización de prácticas humanizadas, a su vez la generación de políticas públicas que permitan su implementación, de esta forma lograr caracterizar la atención en salud con una excelsa humanización.

\section{Consideraciones finales:}

A través del presente documento se logró describir los elementos transversales del trabajo de Jean Watson; se puede concluir que es una teoría que cada vez está siendo más utilizada producto de la creciente necesidad de humanizar la atención en salud, según Watson los momentos del cuidado son la esencia de su teoría, ella propone 10 Factores de Cuidados que después trasladó a Proceso Caritas de Cuidados, estos factores son los que enfermería utiliza en la prestación de la atención hacia el paciente, los cuales fueron desarrollados a partir de una filosofía humanística.

Con el paso del tiempo se han descrito copiosas situaciones que denotan deshumanización del cuidado, una lamentable realidad, donde es hora de comenzar a ocuparnos; bajo esa premisa se gesta la propuesta de generar normas o protocolos donde se desarrolle una estandarización de la humanización del cuidado humano, cuyos resultados sean aquilatados a través de indicadores de cuidado humanizados, estos últimos ya han sido proporcionados por la comunidad científica. 


\section{> Referencias bibliográficas:}

1. d'Empaire G. Calidad de atención médica y principios $\sqrt{\odot}$ ticos. Acta bioeth. [Internet] 2010 Nov [consultado 23 Nov 2020];16(2): 124-132.

Disponible en: https://bit.ly/3piqjmH

2. Ley $\mathrm{N}^{\circ} 20.584$ que regula los derechos y deberes que tienen las personas en relación con acciones vinculadas a su atención en salud. Diario Oficial de Chile, 24 Abr 2012 [consultado 24 Nov 2020]. Disponible en: https://bit.ly/3DddleQ

3. Cabrera M. Usuarios de urgencia maternal de Hospital de Puerto Montt denuncian mala atención y maltratos. Biobiochile.cl [Internet]. 2019 [citado 15 junio 2020]. Disponible en: https://bit. ly/3DddleQ

4. Briones N. Mujer acusa malos tratos en Cesfam de Temuco: reclamó por larga espera y llamaron a Carabineros. Biobiochile.cl [Internet]. 2018 [citado 15 junio 2020]. Disponible en: https:// bit.ly/3of8428

5. Leiva L, Herrera J. Uno de cada cuatro reclamos en hospitales y consultorios públicos es por maltrato. Latercera.com [Internet]. 2016 [citado 15 junio 2020]. Disponible en: https:// bit.ly/3d9mGtM

6. TV5. Denuncian malos tratos a niño con autismo en Cesfam de Calle Larga. TV5 [Internet]. 2021 [citado 06 octubre 2021]. Disponible en: https:// bit.ly/3ltQJAG

7. Stuardo M. "Ustedes no se vacunan": acusan malos tratos a pareja de adultos mayores en cesfam de Concepción. Biobiochile.cl [Internet]. 2021 [citado 06 octubre 2021]. Disponible en: https:// bit.ly/3GbeHsK

8. Chvnoticias. "Decide no operarme hasta que llegue el cheque en garantía": doctor deberá pagar millonaria indemnización. Chvnoticias [Internet]. 2020 [citado 06 octubre 2021]. Disponible en: https://bit.ly/3xQyf2n

9. Chvnoticias. Joven denunció negligencia médica tras ser operado del pie equivocado. Chvnoticias [Internet]. 2017 [citado 06 octubre 2021]. Disponible en: https://www.chvnoticias.cl/ nacional/joven-denuncio-negligencia-medicatras-ser-operado-del-pie-equivocado_20171018/

10. Mennickent C. "Dijeron que me había cagado la vida": violencia obstétrica. Biobiochile.cl [Internet]. 2021 [citado 06 octubre 2021]. Disponible en: https:// bit.ly/3odP9VC

11. Universidad María Auxiliadora. Dra. Jean Watson en la UMA [Video File]. Fecha 2016, agosto 9. [citado 8 junio 2020] [25:39 min.]. Disponible en: https:// bit.ly/3xKThzD

12. Goic A. El Sistema de Salud de Chile: una tarea pendiente. Rev. méd. Chile. 2015 Jun [citado 23 Nov 2020]; 143(6): 774-786. Disponible en: https:// bit.ly/3xMRiup

13. Watson Caring Science [Internet]. Colorado: Jean Watson; 17 diciembre, 2019 [citado 2 de junio 2020]. Watson Caring Science Institute; [aprox. 3 p.]. Disponible en: https://bit.ly/3pkZMp1

14. Alligood M. Nursing theorists and their work. 9th ed. St. Louis, MO: Elsevier Inc; 2018. ISBN: 978-0-323-40224-8

15. Watson J. The philosophy and science of human caring. 1st ed. Boston: Little, Brown; 1979.

16. Watson J. Nursing: The Philosophy and Science of Caring. Revised Edition. Boulder, Colorado: University Press of Colorado; 2008.

17. Colchón A, Núñez O. Cuidado Humano: saber, ser y hacer del estudiante de enfermería, según la teoría de Jean Watson en una Universidad 
Nacional de Lambayeque 2016 - 2017 [Licenciado]. Universidad Nacional Pedro Ruiz Gallo; 2018.

18. Marriner A, Raile M. Modelos y Teorías de Enfermería. 8va ed. Elsevier. España: 2009.

19. Watson, J. Nursing: The Philosophy and Science of Caring. Boulder: Colorado University; 1985.

20. Serrato D. Cuidado humanizado desde la perspectiva del profesional de enfermería del hospital provincial docente Belén Lambayeque- 2017 [Licenciado]. Universidad Señor de Sipán; 2019.

21. Watson J. Watson's theory of human caring and subjective living experiences: carative factors/caritas processes as a disciplinary guide to the professional nursing practice. Texto contexto - enferm. [Internet]. 2007 Mar [citado 15 Oct 2020];16(1):129-135. Disponible en: http://www.scielo.br/scielo.php?script=sci_arttext \&pid=S010407072007000100016\&lng=en.

22. Watson J. Human Caring Science, A theory of nursing. 2a. Ed, Boston: Jones and Bartlett Publisher, 2012.

23. Pajnkihar M, McKenna H, Štiglic G, Vrbnjak D. Fit for Practice: Analysis and Evaluation of Watson's Theory of Human Caring. Nursing Science Quarterly. 2017 [citado 15 Oct 2020];30(3):243-252. https://doi.org/ 10.1177 / 0894318417708409

24. Watson J. Caring science as sacred science. Philadelphia: F.A. Davis; 2005.

25. Watson J. Nursing: human science and human care: a theory of nursing. Boston: Jones and Bartlett; 1999.

26. Watson J. Enfermería: ciencia humana y cuidado humano. Una teoría de enfermería. Segunda impresión. Nueva York: National League for Nursing Publications; 1988.
27. Cusinga F, Mejia F, Obeso L. Aplicación de la Teoría de Enfermería de Jean Watson y la Calidad del Cuidado Enfermero de la Unidad de Cuidados Intensivos Pediátrica del Instituto Nacional de Salud del Niño, Lima, 2017 [Enfermería en cuidados intensivos pediátricos]. Universidad Peruana Unión; 2017.

28. Hermosilla A, Mendoza R, Contreras S. Instrumento para valoración del cuidado humanizado brindado por profesionales de enfermería a personas hospitalizadas. Index Enferm. 2016 Dic [citado 10 Jun 2020]; 25(4): 273-277. Disponible en: http://scielo.isciii.es/scielo.php?script=sci_ arttext\&pid=S1132-12962016000300011\&lng=es.

29. Durgun Y. Implementation of Watson's Theory of Human Caring: A Case Study. Texto completo - enferm. [Internet]. January-April 2015 [citado 10 Jun 2020]; pag. 25-35. Disponible en: http:// www.internationaljournalofcaringsciences.org/ docs/4-Lash\%20-\%200riginal.pdf

30. Peña S, Flórez J, Calvache I, Molina L. Repercusión del uso del Celular en el Cuidado de Enfermería mediante la Teoría del Cuidado Humano de Jean Watson. RCR [Internet]. 14 de septiembre de 2018 [citado 12 Jun 2020];2(2):30-9. Disponible en: https://journals.uninavarra.edu.co/index.php/ cinaresearch/article/view/132

31. Guerrero-Ramírez $\mathrm{R}$, Meneses-La Riva $\mathrm{M}$, De La Cruz-Ruiz M. Cuidado humanizado de enfermería según la teoría de Jean Watson, servicio de medicina del Hospital Daniel Alcides Carrión. Lima- Callao, 2015. Revista Enfermería Herediana. 2016 [citado 13 Nov 2020];9(2):127-136. Disponible en: https://pdfs.semanticscholar.org/887e/ f02 bf5547abb50cd1c46c4be 5252 b 1 b 89 f 95 . pdf?_ga=2.189894059.1427057129.16335447291712750862.1633544729

32. Hubert P. Application of Jean Watson's 
Theory of Transpersonal Caring in Nurses Practicing in a Pain Center [Doctor of Nursing Practice]. Universidad de Seton Hall; 2018.

33. Riegel F, Crossetti M, Siqueira D. Contributions of Jean Watson's theory to holistic critical thinking of nurses. Rev. Bras. Enferm. [Internet]. 2018 Aug [citado 10 Jun 2020];71(4): 2072-2076. Disponible en: https://doi.org/10.1590/0034-71672017-0065.

34. McMillan M. The Effects of Watson's Theory of Human Caring on the Nurse Perception and Utilization of Caring Attributes and the Impact on Nurse Communication [Doctor of Nursing Practice]. Gardner-Webb University; 2017.

35. Santos K. O Cuidar em Enfermagem a Pessoa com Anemia Falciforme: Aplicação da Teoria de Jean Watson na Relação EnfermeiraIndivíduo. Saúde em Revista. 2016 [citado 15 Oct 2020];16(44):55-61. Disponible en: http://dx.doi. org/10.15600/2238-1244/sr.v16n44p55-61

36. Do Nascimento B, De Sousa T, Santos K, Schober V, Sobral da Silva J, Szalbot J et al. Aplicação da teoria do cuidado humano em uma unidade de terapia intensiva: relato de experiência da enfermagem. Revista Destaques Acadêmicos. 2019 [citado 17 Oct 2020];11(3):59-70. Disponible en: http://dx.doi.org/10.22410/issn.2176-3070. v11i3a2019.2116.

37. Seaward B, Lissard C. A Spiritual WellBeing Model for the Healing Arts. Journal of Holistic Nursing. 2020 [citado 19 Oct 2020];38(1):102106. Disponible en: https://doi.org/10.1177 / 0898010120907528

38. Watson Caring Science Institute. Investigación y herramientas de emedición de la ciencia del cuidado. 2021. Obtenido el 10 junio de 2021, a partir de: https://www.watsoncaringscience.org/jean- bio/caring-science-theory/research/watson-caritas-patient-score/

39. Brewer B, Anderson J, Watson J. Evaluación de cambios en los comportamientos de cuidado de los entrenadores de Caritas antes y después del programa de educación para entrenadores de Caritas, JONA: The Journal of Nursing Administration. 2020 [citado 20 Oct 2020]; 50(2):85-89. Disponible en: https://pubmed.ncbi.nlm.nih.gov/31977945/

40. Ministerio de Salud. Repositorio bibliográfico y patrimonial

del ministerio de salud de Chile. MINSAL [Internet]. 2021 [citado 14 octubre 2021]. Disponible en: http://www.repositoriodigital.minsal.cl/handle/2015/8

41. Melita R. Indicadores de cuidado humanizado de enfermería en hospitales de la región del Bio Bío, Chile [Doctor]. Universidad de Concepción; 2018. 